\title{
Evaluating the effect of Zinc Oxide nanoparticles doped with Gadolinium on dose enhancement factor by PRESAGE dosimeter
}

\author{
N. Banaee', H.A. Nedaie ${ }^{2,3}{ }^{*}$, A.R. Shirazi3, A.R. Zirak4, S. Sadjadi5 \\ ${ }^{1}$ Department of Engineering, Science and Research Branch, Islamic Azad University, Tehran, Iran \\ ${ }^{2}$ Radiotherapy Oncology Department, Cancer Research Center, Cancer Institute, Tehran University of Medical \\ Sciences, Tehran, Iran \\ ${ }^{3}$ Department of Medical Physics and Biomedical Engineering, Faculty of Medicine and Radiation Oncology \\ Research Centre, Cancer Institute, Tehran University of Medical Sciences, Tehran, Iran \\ ${ }^{4}$ Laser and Optics Research School, NSTRI, Tehran, Iran \\ ${ }_{5}^{5}$ Nuclear Science and Technology Research Institute, Tehran, Iran
}

\section{- Original article}

\author{
*Corresponding author: \\ Dr. Hassan Ali Nedaie, \\ Fax: +9821 66948673 \\ E-mail: \\ Nedaieha@sina.tums.ac.ir \\ Revised: June 2015 \\ Accepted: July 2015 \\ Int. J. Radiat. Res., April 2016; \\ 14(2): 119-125 \\ DOI: 10.18869 /acadpub.ijrr.14.2.119
}

\begin{abstract}
Background: New treatment modalities are developed with the aim of escalating tumor absorbed dose and simultaneously sparing the normal structures. The use of nanotechnology in cancer treatment offers some possibilities including destroying cancer tumors with minimal damage to healthy tissues. Zinc Oxide nanoparticles ( $\mathrm{n}$ O NPs) are wide band gap semiconductors and seem to have a good effect on increasing the absorbed dose of target volume especially when doped with a high $Z$ element. The aim of this study was to evaluate the effect of ZnO NPs doped with Gadolinium (Gd) on dose enhancement factor by 6MV photon beam irradiation. Materials and Methods: Various concentrations of ZnO NPs doped with $5 \%$ Gd were incorporated into PRESAGE composition, the 3D chemical dosimeter. Then by using a UV-Vis spectrophotometer optical density changes and also dose enhancement factor (DEF) were determined. Results: The results of this study showed that by incorporating 500, 1000, 3000 and $4000 \mu \mathrm{g} / \mathrm{ml} \mathrm{ZnO} \mathrm{NPs}$ doped with Gd into PRESAGE structure the dose enhancement factor of about 1.57, 1.69, 1.78 and 1.82 in a $15 \times 15 \mathrm{~cm}^{2}$ field size could be found, respectively. Conclusion: The results of this study showed that ZnO NPs doped with Gd could be considered as new compound for increasing the absorbed dose.
\end{abstract}

Keywords: Zinc Oxide, Gadolinium, Nanoparticle, PRESAGE, DEF.

\section{INTRODUCTION}

Advanced treatment delivery techniques such as Three Dimensional Radiation Therapy (3DCRT), Intensity Modulated Radiation Therapy (IMRT), Stereotactic Radio Surgery (SRS), Stereotactic Radio Therapy (SRT) and Volumetric Modulated Arc Therapy (VMAT) are currently used with the ultimate goal of conforming the radiation dose in such a way that delivers maximum lethal dose to the target volume whilst minimizing doses to organs at risk $(1,2)$. These methods of treatment improve the therapeutic ratio and enable dose escalation in the target volume, hence reducing the radiation related complications and consequently cause the NTCP (normal tissue complication probability) and TCP (tumor control probability) curves to have a more disunion from each other $(3,4)$.

The use of nanotechnology in cancer treatment offers some exciting possibilities including the opportunity of destroying tumors with lower doses and consequently lower 
damage to healthy tissues (5-13). There have been done several studies focusing on importance and influence of nanoparticles on increasing therapeutic ratio and also dose enhancement on irradiated volume especially by Gold nanoparticles $(14,15)$. Among nanoscience, Zinc Oxide nanoparticles (ZnO NPs) are interesting wide band gap semiconductors $(3.37 \mathrm{eV})$, capable of producing reactive oxygen species (ROS) and oxygen free radicals. ROS generation is linked to cell damage and cellular apoptosis (13, 16-18).

It is evident that high $\mathrm{Z}$ materials absorb Xray significantly. Therefore, it is hypothesized that the interaction of ZnO NPs with X-rays could be optimized by the addition of rare earth (RE) elements as dopants. The probability of an X-ray photon being absorbed or scattered by a particular material is a function of photon interaction cross-section. A greatly enhanced photoelectric cross-section can be derived from the introduction of a material with a high atomic number (Z). If a high $\mathrm{Z}$ element could be readily and specifically incorporated into cells and then exposed to photons of energy just above the binding energy of the innermost orbital (K edge), a selective absorption might be induced. One such element that has precedent in medical diagnostics is Gadolinium (Gd) which has an $\mathrm{X}$-ray photon interaction cross-section significantly greater than the elements that comprise biological tissues (19-21). It seems that ZnO NPs doped with Gd have the potential to increase the total absorbed dose of irradiated volume and consequently not only lead to spare the surrounding normal tissues but also reduces the needed Monitor Unite (MU) to deliver that amount of dose. Protocols for these complex treatment delivery techniques have been developed to involve very steep dose gradients and are therefore, extremely sensitive to errors in treatment delivery. To minimize such errors, 3D dosimeters were developed as a relative method for improving dose monitoring and delivery ${ }^{(1,2)}$.

One of the most significant developments in 3D dosimetry over the past decade was the introduction of radiochromic dosimeter (PRESAGE) (22-25). PRESAGE dosimeter is not considered as a gel dosimeter, but rather a clear polyurethane resin containing radiationsensitive reporter components (leuco dye) and halogenated carbon radical initiators. Free radicals result from the homolysis of the bond between carbon and the halogen upon irradiation. These radicals oxidize the leuco dye that is also incorporated in the formulation, leading to a change in color (optical density). The change in optical density is linear with respect to the absorbed radiation dose. According to previous studies, this type of dosimeter has the potential to be used in clinical dose measurements (22-25).

The aim of this study was to determine the dose enhancement factor of ZnO NPs doped with Gd as a novel nanoscale composition, by use of PRESAGE dosimeter, irradiating by $6 \mathrm{MV}$ photon beams.

\section{MATERIALS AND METHODS}

\section{PRESAGE fabrication}

At first, PRESAGE dosimeter was fabricated. The procedure of PRESAGE fabrication was as follows:

(i) Free radical initiator and reporter compound were thoroughly mixed. The chosen radical initiator and reporter compound were $\mathrm{CCl}_{4}$ (Merck, Keinlworth, United States) and leucomalachite green (LMG) (Sigma Aldrich, St Louis, MO, United States), respectively. (ii) Polyurethane resin (Crystal Clear 2006, SmoothOn, Easton, PA, USA) was supplied in two parts (Part A and Part B). These compounds were mixed together to afford optically clear polyurethane resins that form the matrix of the PRESAGE dosimeter. (iii) The solutions prepared in steps (i) and (ii) were combined together and thoroughly mixed. (iv) The mixture prepared in step (iii) was poured into poly spectrophotometer cuvettes and the filled cuvettes were placed under certain pressure for a few days to minimize out gassing (22-25).

Polyurethane is approximately water equivalent but according to Mayneord formula, adding radical initiators and leuco dye cause the effective atomic number to be greater than that 
of water. Equation 1 shows Mayneord formula:

$$
Z_{\text {eff }}=\sqrt[2.94]{f_{1} \times\left(Z_{1}\right)^{2.94}+f_{2} \times\left(Z_{2}\right)^{2.94}+f_{3} \times\left(Z_{3}\right)^{2.94}+\ldots}
$$

In which, $f_{n}$ is the fraction of the total number of electrons associated with each element, and $Z_{n}$ is the atomic number of each element (24).

In general, dosimetric properties of PRESAGE are affected by the fractional weight of its elements (22-26). Therefore, the percents of components need to be arranged and calculated in such a way to be water equivalent.

In order to prepare an almost water equivalent dosimeter and also save other good dosimetric properties of PRESAGE including sensitivity, linearity, stability and etc. percentage of PRESAGE composition was adjusted according to the study done by Mostaar et al. (24).

By obtaining the chemical formulations of PRESAGE components and using Mayneord formula, the effective atomic number of fabricated dosimeter would be 7.8.

\section{Read-out wavelength}

At second step to determine the maximum absorption wavelength of PRESAGE, the absorption spectrum of PRESAGE filled cuvettes over the visible wavelength $(470-750 \mathrm{~nm})$ were obtained by use of a UV-Vis spectrophotometer (Varian, Palo Alto, California, USA). Then the maximum absorption of each cuvette was obtained pre irradiation.

\section{Calibration}

The next process was calibration of PRESAGE against ionization chamber (Farmer chamber, $0.6 \mathrm{cc}$, PTW, Freiburg, Germany) to deliver definite steps of absorbed dose. Cuvettes were placed in a solid water phantom made by Plexiglas (figure 1) and irradiated by $15 \times 15 \mathrm{~cm}^{2}$ field to deliver the absorbed doses including 0 , $0.5,1,2,4,6,8,10,12,14,16,18,20$ Gy irradiated by $6 \mathrm{MV}$ photon beams producing by a linear accelerator (Varian Clinac 2100 C). Various slab phantoms were used to prepare the electronic equilibrium condition (figure 2) (27). For each dose step, three cuvettes were used. All cuvettes were kept in dark and cold

environment to prevent any accidental absorption change and 48 hours after irradiation, the maximum absorption of each cuvette was obtained by using UV-Vis spectrophotometer, similar to pre irradiation phase. Finally, by subtracting the pre irradiation absorbance values from that of post irradiation, calibration curve which is the variations of optical density against absorbed dose, was plotted (28).

\section{Nanoparticle synthesization}

At the next section, $\mathrm{ZnO}$ NPs and $5 \% \mathrm{Gd}$ doped NPs were prepared and incorporated in to the composition of the PRESAGE and the irradiation processes were repeated.

Nanoparticles were prepared by chemical precipitation route using Zinc acetate, sodium hydroxide, gadolinium nitrate and absolute ethanol.

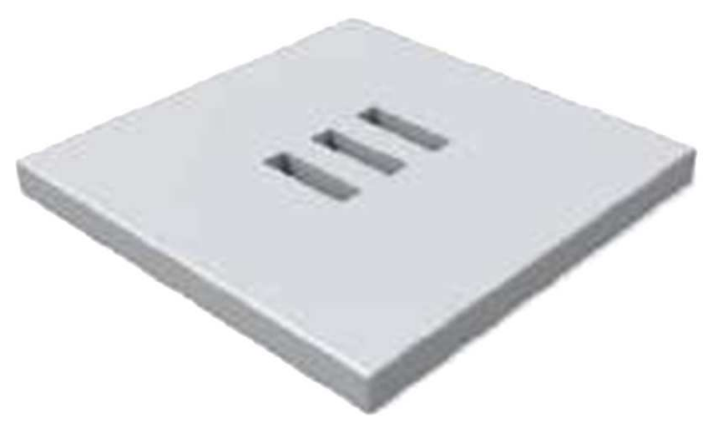

Figure 1. A designed slab phantom .

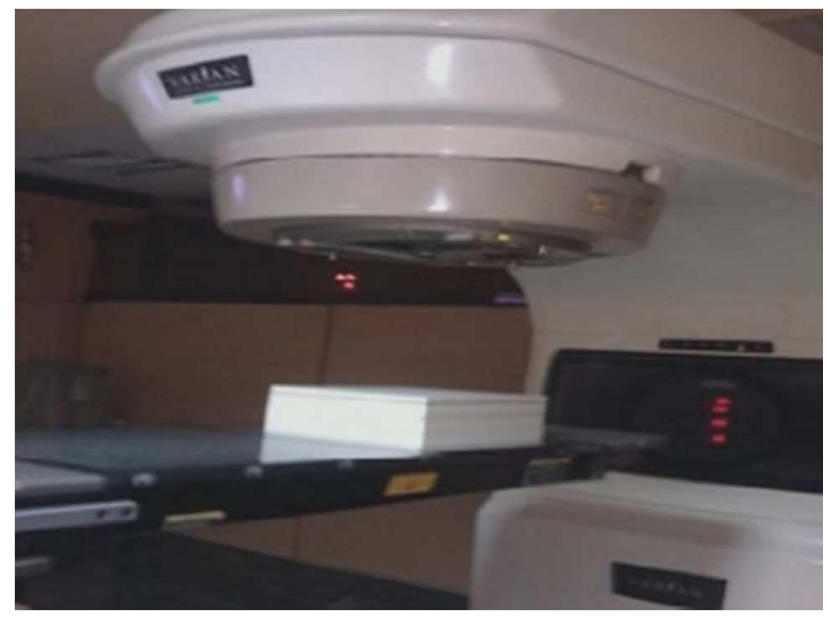

Figure 2. Setup of calibration process.

Int. J. Radiat. Res., Vol. 14 No. 2, April 2016 
Zinc acetate dehydrate (0.2195 g) was dissolved in $20 \mathrm{ml}$ of absolute ethanol and stirred with a magnetic stirrer at room temperature, to be mixed thoroughly. Then the solution of $\mathrm{NaOH}(0.08 \mathrm{~g} \mathrm{NaOH}$ in $20 \mathrm{ml}$ of absolute ethanol) was added dropwise into the solution of Zinc acetate under constant magnetic stirring for $1 \mathrm{~h}$. After that $0.015 \mathrm{mmol}$ of oleic acid was added into the solution. The white powder was obtained after stirring this solution at room temperature for another $1 \mathrm{~h}$. The prepared dispersion was centrifugally filtered and washed with ethanol and distilled water for several times, followed by drying in an oven at $50{ }^{\circ} \mathrm{C}$ for $4 \mathrm{~h}(29)$.

For doped $\mathrm{ZnO}$ nanoparticles the appropriate amount of gadolinium nitrate $(5 \% \mathrm{w} / \mathrm{w}$ Zinc acetate dehydrate) was added in the homogenized and clear solution of Zinc acetate dehydrate and ethanol. The remaining procedures were the same as described above.

\section{Incorporating NPs into PRESAGE}

For determining the effects fabricated NPs on $\mathrm{DEF}$, various concentrations of $\mathrm{ZnO} \mathrm{NPs}$ and $\mathrm{ZnO}$ NPs doped with Gd were incorporated in to PRESAGE composition and irradiation and read out procedures were repeated.

\section{Comparing the results}

Finally, by applying descriptive statistics, the results of dosimetry at presence and lack of NPs were compared and dose enhancement factor was determined.

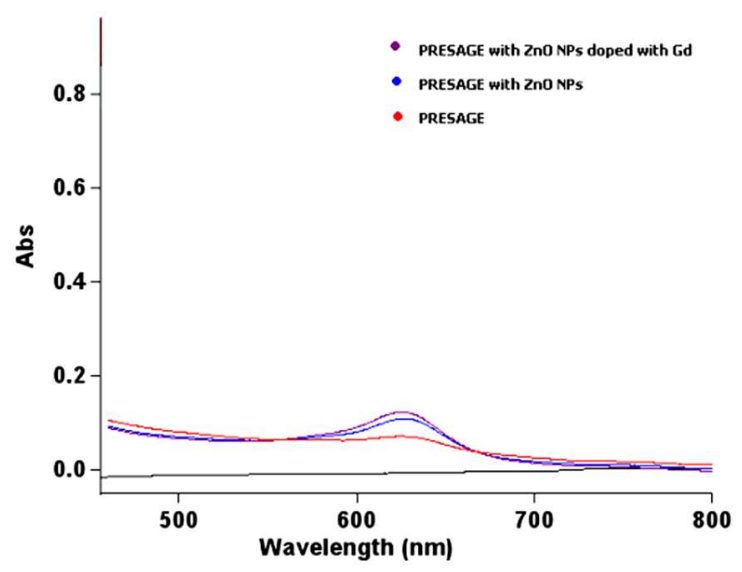

Figure 3. The absorption spectrum of various compositions of PRESAGE over visible wavelengths.

\section{RESULTS}

\section{Read-out wavelength}

Figure 3 depicts the absorption spectrum of different compositions of PRESAGE (with and without NPs) over visible wavelength. As it can be seen, the maximum absorption of all series of PRESAGE occurred at about $632 \mathrm{~nm}$.

\section{Calibration}

Figure 4 represents the optical density changes of PRESAGE against various dose steps.

\section{DEF determination}

Dose enhancement factor (DEF) is defined as the ratio of changes in optical density with NPs over the optical density changes without NPs (14). Since PRESAGE exhibits a linear behavior against various dose levels (figure 4), comparing the optical density changes of all series of PRESAGEs in a particular dose step is feasible.

Figure 5 shows the PRESAGE filled cuvettes with and without NPs, delivered a dose of $10 \mathrm{~Gy}$. The color changes caused by presence of NPs are obvious.

Table 1 shows the optical density changes of all fabricated PRESAGEs irradiated by $6 \mathrm{MV}$ photon beams and 10 Gy delivered radiation dose. According to definition of DEF, this parameter was acquired for different compositions of PRESAGE and tabulated in table 2. Comparisons of DEF for various concentrations of applied NPs are depicted in figure 6 .

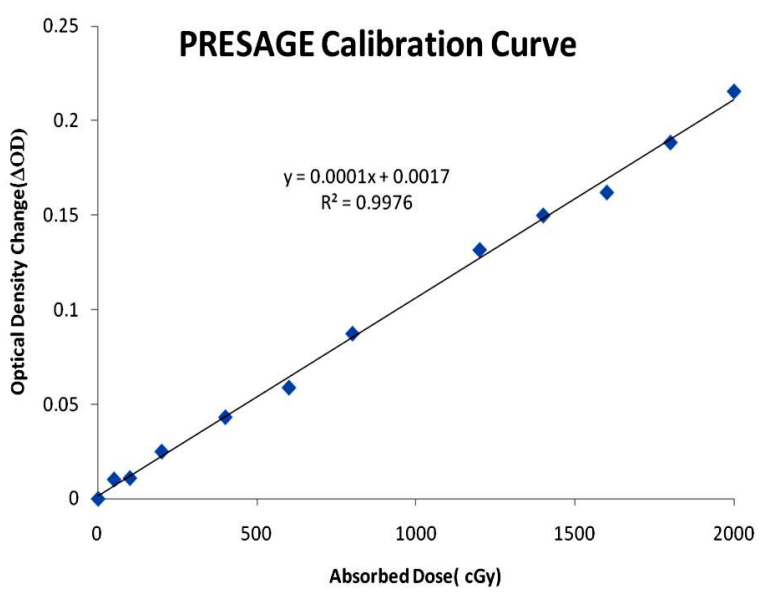

Figure 4. Calibration curve of PRESAGE. 


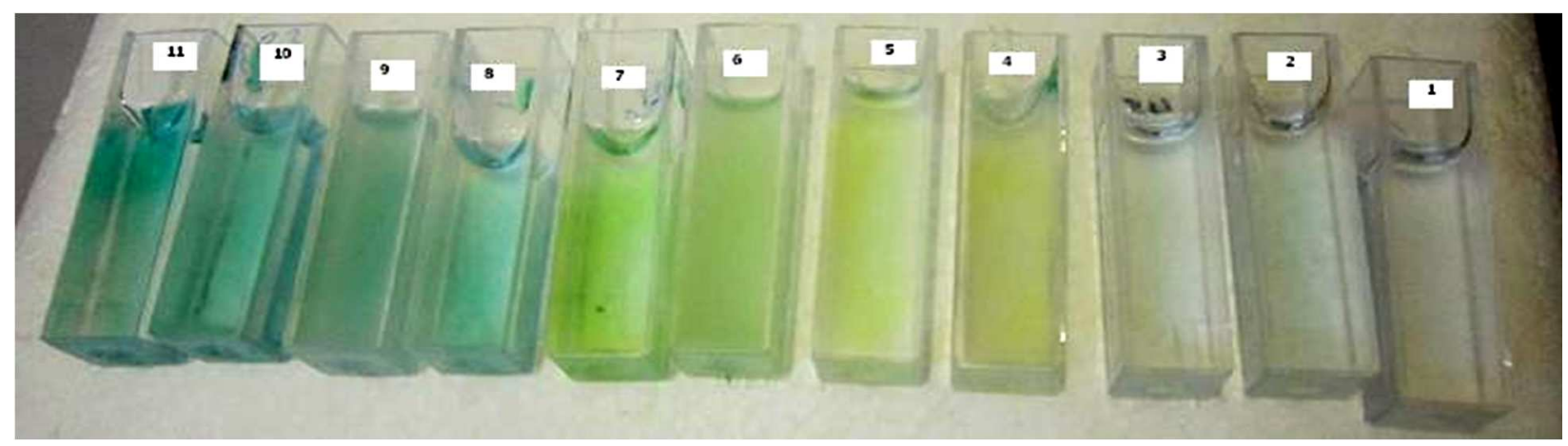

Figure 5. PRESAGE filled cuvettes with and without NPs at dose of 10Gy. 1) PRESAGE without NPs. 2) PRESAGE with 20 $\mu \mathrm{g} / \mathrm{ml}$ ZnO NPs. 3) PRESAGE with $20 \mu \mathrm{g} / \mathrm{ml}$ ZnO NPs doped with $\% 5$ Gd. 4) PRESAGE with $500 \mu \mathrm{g} / \mathrm{ml} \mathrm{ZnO}$ NPs. 5) PRESAGE with $500 \mu \mathrm{g} / \mathrm{ml}$ ZnO NPs doped with $\% 5$ Gd. 6) PRESAGE with $1000 \mu \mathrm{g} / \mathrm{ml}$ ZnO NPs. 7) PRESAGE with $1000 \mu \mathrm{g} / \mathrm{ml}$ ZnO NPs doped with \%5 Gd. 8) PRESAGE with $3000 \mu \mathrm{g} / \mathrm{ml}$ ZnO NPs. 9) PRESAGE with $3000 \mu \mathrm{g} / \mathrm{ml}$ ZnO NPs doped with $\% 5 \mathrm{Gd}$. 10) PRESAGE with $4000 \mu \mathrm{g} / \mathrm{ml} \mathrm{ZnO}$ NPs. 11) PRESAGE with $4000 \mu \mathrm{g} / \mathrm{ml} \mathrm{ZnO}$ NPs doped with $\% 5 \mathrm{Gd}$.

Table1. Optical density changes of various PRESAGE compositions.

\begin{tabular}{|c|c|c|}
\hline $\begin{array}{c}\text { Concentration } \\
\text { of NPs }(\mu \mathrm{g} / \mathrm{ml})\end{array}$ & Added Composition & $\begin{array}{c}\text { Optical Density } \\
\text { Changes }\end{array}$ \\
\hline------ & PRESAGE & 0.1095 \\
\hline 20 & $\begin{array}{c}\text { ZnO NPs doped with 5\% Gd } \\
\text { ZnO NPs }\end{array}$ & $\begin{array}{l}0.1099 \\
0.1096\end{array}$ \\
\hline $\mathbf{5 0 0}$ & ZnO doped with \%5 Gd & 0.1719 \\
& ZnO NPs & 0.1445 \\
\hline $\mathbf{1 0 0 0}$ & ZnO NPs doped with 5\% Gd & 0.1850 \\
& ZnO NPs & 0.1664 \\
\hline $\mathbf{3 0 0 0}$ & ZnO doped with \%5 Gd & 0.1945 \\
& ZnO NPs & 0.1741 \\
\hline $\mathbf{4 0 0 0}$ & ZnO NPs doped with 5\% Gd & 0.1997 \\
& ZnO NPs & 0.1807 \\
\hline
\end{tabular}

Table 2. DEF of different compositions of PRESAGE.

\begin{tabular}{|c|c|c|}
\hline $\begin{array}{c}\text { Concentration } \\
\text { of NPs }(\mu \mathrm{g} / \mathrm{ml})\end{array}$ & Def \\
\hline $\mathbf{2 0}$ & ZnO NPs doped with $5 \% \mathrm{Gd}$ & 1.004 \\
& ZnO NPs & 1.001 \\
\hline \multirow{2}{500}{} & ZnO doped with \%5 Gd & 1.57 \\
& ZnO NPs & 1.32 \\
\hline \multirow{2}{*}{$\mathbf{0 0 0 0}$} & ZnO NPs doped with 5\% Gd & 1.69 \\
& ZnO NPs & 1.52 \\
\hline \multirow{2}{*}{$\mathbf{3 0 0 0}$} & ZnO doped with \%5 Gd & 1.78 \\
& ZnO NPs & 1.59 \\
\hline
\end{tabular}

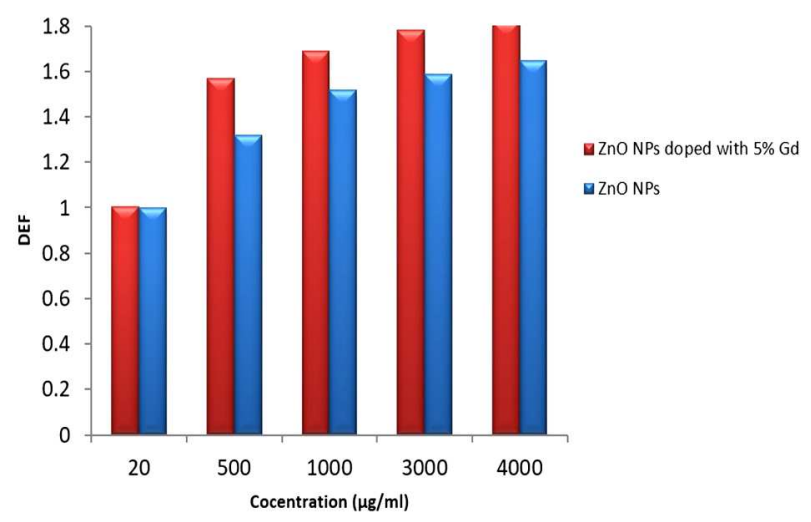

Figure 6. Comparisons of DEF for various concentrations of NPs.

\section{DISCUSSION}

High Z materials absorb X-ray significantly and $\mathrm{Gd}$ is a high $\mathrm{Z}$ element which can increase the probability of photoelectric effect when irradiated by photons $(19,20)$. Although the probability of photoelectric effect for $6 \mathrm{MV}$ photon beams is low compared to orthovoltage beams, but regarding to the continues X-ray spectrum, attenuated photons, low energy photon beams in X-ray spectrum (30) and probably the pair production effect, DEF is clearly observed.

As it is shown in table 2 and figure 6 , the maximum DEF is acquired by applying $4000 \mu \mathrm{g} /$ $\mathrm{ml} \mathrm{ZnO} \mathrm{NPs} \mathrm{doped} \mathrm{with} \mathrm{Gd.} \mathrm{It} \mathrm{is} \mathrm{also} \mathrm{shown} \mathrm{that}$ small concentration of NPs $(20 \mu \mathrm{g} / \mathrm{ml})$ does not have a significant role in DEF even if it was doped by a high $\mathrm{Z}$ element such as Gd. 
For delivering an absorbed dose of $10 \mathrm{~Gy}$, an almost 995 MU was needed. Since by using $4000 \mu \mathrm{g} / \mathrm{ml} \mathrm{ZnO} \mathrm{NPs,} \mathrm{for} \mathrm{delivering} \mathrm{the} \mathrm{same}$ absorbed dose, the needed MU would be below 600. This achievement can lead to decrease treatment time and therefore, treatment time reduction generally causes less patient movement during treatment and consequently random errors of treatment would dwindle which are the benefits of NPs application.

It is noteworthy to mention that $\mathrm{Gd}$ is a toxic element and toxicity is usually transferred from the surface of element (31). Therefore, for increasing the absorbed dose it not feasible to use Gd without any surrounded structure. In other words, by doping the Gd into the Zinc Oxide NPs' crystal, Gd toxicity releasing to the neighboring atoms is omitted.

Type, size, concentration of nanoparticles and also the quality of photon beams are the items which have significant influence on DEF. Since there was not any study about the application of ZnO NPs with a high Z dopant as dose enhancing substance for megavoltage photon beams, it is not possible to make a comparison.

\section{CONCLUSION}

The results of this study revealed that $\mathrm{ZnO}$ NPs doped with Gd are new proposing substances for enhancing the absorbed dose and increasing the therapeutic ratio even in high energy photon beams. Various reasons may cause the DEF for 6MV photon beams such as photoelectric effect for low energy photon beams in continues X-ray spectrum, attenuated photons or pair production effect. Using these NPs not only reduces the needed MU to deliver a certain amount of absorbed dose, but also can lead to great succeeds in reducing treatment time and random errors in treatment procedure.

\section{Conflict of interest: Declared none.}

\section{REFERENCES}

1. Mayles P, Nahum A, Rosenwald JC (2007) Handbook of radiotherapy physics: theory and practice. Boca Raton (FL),

Int. J. Radiat. Res., Vol. 14 No. 2, April 2016
Taylor and Francis Group.

2. BaskarR, Lee K A, YeoR, YeohK W (2012) Cancer and radiation therapy: current advances and future directions. Int J Med Sci, 9:193-199.

3. Khan F M and Gerbi B J (2005) Treatment planning in radiation oncology. Lippincott Williams \& Wilkins.

4. Connell P P and Hellman S (2009) Advances in Radiotherapy and Implications for the Next Century: A Historical Perspective. Cancer Res, 69:383-392.

5. Mesbahi A, Jamali F, Gharehaghaji N (2013) Effect of Photon Beam Energy, Gold Nanoparticle Size and Concentration on the Dose Enhancement in Radiation Therapy. Biolmpacts, 3: 29-35.

6. Némati $F$, Dubernet $C$, Fessi $H$, Colin de Verdière $A$, Poupon M F, Puisieux $F$ et al. (1996) Reversion of multidrug resistance using nanoparticles in vitro: Influence of the nature of the polymer. Int J Pharm, 138: 237-246.

7. Bahreyni Toossi M T, Ghorbani M, Sobhkhiz Sabet L, Akbari F, Mehrpouyan M (2015) A Monte Carlo study on dose enhancement and photon contamination production by various nanoparticles in electron mode of a medical linac. NUKLEONIKA, 60:489-496.

8. Geso M (2012) Nanoparticle augmented radiation treatment decreases cancer cell proliferation. Nanomedicine: Nanotechnology, Biology and Medicine, 8: 526-536.

9. Schilling K, Bradford B, Castelli D, Dufour E, Nash JF, Pape W et al. (2010) Human safety review of "nano" titanium dioxide and zinc oxide. Photochem Photobiol Sci, 9:495509.

10. Liong M, Lu J, Kovochich M, Xia T, Ruehm SG, Nel AE et al. (2008) Multifunctional inorganic nanoparticles for imaging, targeting, and drug delivery. ACS Nano, 2:889-96.

11. Li XH, Xing YG, Li WL, Jiang YH, Ding YL (2010) Antibacterial and physical properties of poly(vinyl chloride)-based film coated with $\mathrm{ZnO}$ nanoparticles. Food Sci Technol Int, 163:225-32.

12. Moos PJ, Chung K, Woessner D, Honeggar $M$, Cutler NS, Veranth JM (2010) ZnO particulate matter requires cell contact for toxicity in human colon cancer cells. Chem Res Toxicol, 23(4):733-9.

13. Franklin NM, Rogers NJ, Apte SC, Batley GE, Gadd GE, Casey PS (2007) Comparative toxicity of nanoparticulate $\mathrm{ZnO}$, bulk $\mathrm{ZnO}$, and $\mathrm{ZnCl} 2$ to a freshwater microalga (Pseudokirchneriella subcapitata): the importance of particle solubility. Environ Sci Technol, 41:8484-90.

14. Mousavie Anijdan S H, Shirazi A, Mahdavi S R, Ezzati A, Mofid B, Khoei S et al. (2012) Megavoltage Dose enhancement of gold nanoparticles for different geometric set-ups: Measurements and Monte Carlo simulation. Iran. J. Radiat. Res, 10: 183-186.

15. Khadem-Abolfazli M, Mahdavi M, Mahdavi S R M, Ataei Gh (2013) Dose enhancement effect of gold nanoparticles on MAGICA polymer gel in mega voltage radiation therapy. Int. J. Radiat. Res. 11: 55-61.

16. Mills A, Lee S K (2002) A web-based overview of semiconductor photochemistry-based current commercial 


\section{Banaee et al. / Dose enhancement factor of ZnO NPs doped}

applications. Journal of Photochemistry and Photobiology A: Chemistry, 152: 233-247

17. Sharma V, Anderson D, Dhawan A (2012) Zinc oxide nanoparticles induce oxidative DNA damage and ROStriggered mitochondria mediated apoptosis in human liver cells (HepG2). Apoptosis, 17: 852-70.

18. Hanley C, Thurber A, Hanna C, Punnoose A, Zhang J, Wingett DG (2009) The Influences of Cell Type and ZnO Nanoparticle Size on Immune Cell Cytotoxicity and Cytokine Induction. Nanoscale Res Lett, 4: 1409-20.

19. Tamura K ,OhkoY ,Kawamura H, Yoshikawa H, Tatsuma T, Fujishima A et al. (2007) X-ray induced photoelectrochemistry on $\mathrm{TiO}_{2}$. Electrochimica Acta, 52: 6938-42.

20. Turner JA (1995) Atoms, Radiation, and Radiation Protection. John Wiley \& Sons, Inc. New York.

21. Villaraza AJ, Bumb A, Brechbiel MW (2010) Macromolecules, dendrimers, and nanomaterials in magnetic resonance imaging: the interplay between size, function, and pharmacokinetics. Chem Rev, 110: 2921-59.

22. Adamovics J and Maryanski M J (2004) A new approach to radiochromic three-dimensional dosimetry-polyurethane. $J$ Phys: Conf Ser, 3: 172-175.

23. Brady SL, Brown WE, Clift CG, Yoo S, Oldham M (2010) Investigation into the feasibility of using PRESAGE/optical$\mathrm{CT}$ dosimetry for the verification of gating treatments. Phys Med Biol. 55: 2187-2201.

24. Clift C, Thomas A, Adamovics J, Chang Z, Das I, Oldham M (2010) Toward acquiring comprehensive radiosurgery field commissioning data using the PRESAGE/optical-CT 3D dosimetry system. Phys Med Biol. 55: 1279-93.

25. Doran S, Al-Nowais S, Krstajić N, Adamovics J, Kacperek A, Brunt J (2006) True-3D scans using PRESAGETM and Optical -CT: A case study in proton therapy. J Phys, Conf Ser, 231234.

26. Mostaar A, Hashemi B, Zahmatkesh M H, Aghamiri S M R, Mahdavi S R (2010) Construction and Development of a Chemical Dosimeter Based on the Radiochromic Solid Polymer and Evaluation of it's Response to High Energy Photons Used in Radiotherapy. J Nucl Sci Tech, 52: 23-29.

27. Banaee $\mathrm{N}$ and Nedaie $\mathrm{H} \mathrm{A}$ (2013) Evaluating the effect of energy on calibration of thermo-luminescent dosimeters 7LiF:Mg,Cu,P (GR-207A). Int J Radiat Res, 11: 51-54.

28. Alqathami M, Adamovics J, Benning R, Qiao G, Geso M, Blencowe $A(2013)$ Evaluation of ultra-sensitive leucomalachite dye derivatives for use in the PRESAGE ${ }^{\circledR}$ dosimeter. Radiat Phys Chem, 85: 204-209.

29. Shouli B, Liangyuan C, Jingwei H, Dianqing L, Ruixian L, Aifan C et al. (2011) Synthesis of quantum size ZnO crystals and their gas sensing properties for NO2. Sensors and Actuators B, 159: 97- 102.

30. Sheihk-Bagheri Dand Rogers D (2002) Monte Carlo calculation of nine megavoltage photon beam spectra using the beam code. Med Phy, 29: 391-402.

31. Vassallo DV, Simões MR, Furieri LB, Fioresi $M$, Fiorim J, Almeida EA, et al. (2011) Toxic effects of mercury, lead and gadolinium on vascular reactivity. Braz J Med Biol Res, 44: 939-46. 
